\title{
Ablation of Arg-tRNA-protein transferases results in defective neural tube development
}

\author{
Eunkyoung Kim ${ }^{1,2}$, Seonmu Kim ${ }^{1}$, Jung Hoon Lee ${ }^{1,2}$, Yong Tae Kwon ${ }^{2}$ E Min Jae Lee Ka, $^{1,3}$ \\ ${ }^{1}$ Department of Biochemistry and Molecular Biology, Seoul National University College of Medicine, ${ }^{2}$ Department of Biomedical Sciences, \\ Seoul National University Graduate School, ${ }^{3}$ Neuroscience Research Institute, Seoul National University College of Medicine, Seoul 03080, \\ Korea
}

The arginylation branch of the $\mathrm{N}$-end rule pathway is a ubiquitin-mediated proteolytic system in which post-translational conjugation of Arg by ATE1-encoded Arg-tRNA-protein transferase to $\mathrm{N}$-terminal Asp, Glu, or oxidized Cys residues generates essential degradation signals. Here, we characterized the $A T E 1^{-1-}$ mice and identified the essential role of $\mathrm{N}$-terminal arginylation in neural tube development. ATE1-null mice showed severe intracerebral hemorrhages and cystic space near the neural tubes. Expression of ATE1 was prominent in the developing brain and spinal cord, and this pattem overlapped with the migration path of neural stem cells. The $A T E 1^{-1-}$ brain showed defective G-protein signaling. Finally, we observed reduced mitosis in $\mathrm{ATE1}^{-1-}$ neuroepithelium and a significantly higher nitric oxide concentration in the $A T E 1^{-l-}$ brain. Our results strongly suggest that the crucial role of ATE1 in neural tube development is directly related to proper turn-over of the RGS4 protein, which participate in the oxygen-sensing mechanism in the cells. [BMB Reports 2016; 49(8): 443-448]

\section{INTRODUCTION}

The $\mathrm{N}$-end rule pathway is one of the substrate recognition mechanisms in the ubiquitin-proteasome system (UPS), which links physiological half-life of a target protein to its $\mathrm{N}$-terminal residue. In the arginylation branch of the $\mathrm{N}$-end rule pathway (Arg/N-end rule pathway), the proteins bearing both a destabilizing $\mathrm{N}$-terminal amino acid and a sterically accessible internal Lys residue(s) (collectively called an $\mathrm{N}$-degron) are generally short-lived (1-3). In mammals, Arg, one of the

${ }^{*}$ Corresponding author. Tel: +82-2-740-8254; Fax: +82-2-7444534; E-mail: minjlee@snu.ac.kr

http://dx.doi.org/10.5483/BMBRep.2016.49.8.087

Received 26 May 2016, Revised 17 June 2016, Accepted 21 June 2016

Keywords: Arginylation, ATE1, G protein signaling, N-end rule, Neural tube primary destabilizing residues, can be attached to $\mathrm{N}$-terminal Asp, Glu, or oxidized Cys by ATE1-encoded Arg-tRNA-protein transferase (Arg-transferase) (4). The newly attached $\mathrm{N}$-terminal Arg residue is recognized and ubiquitinated by a family of E3 ubiquitin ligases named UBR1 through $\operatorname{UBR7}(5,6)$. The $\mathrm{Arg} / \mathrm{N}$-end rule pathway is present even in prokaryotes, which lack ubiquitin (7-9), probably indicating that the $\mathrm{N}$-end rule pathway developed evolutionarily prior to the UPS.

The N-terminal arginylation mediated by ATE1 Argtransferase was identified $\sim 50$ years ago as one of eukaryotic posttranslational modifications (10) and relatively recently was found to play important roles in the control of the half-life of various proteins in the cell. In mammals, two independent papers reported in 2005 that the R4 subfamily of proteinsregulators of $G$ protein signaling (RGS: RGS4, RGS5, and RGS16) are targeted via the ATE1-UBR circuit, and their degradation depends on nitric oxide (NO) and molecular oxygen concentration $(11,12)$, which oxidize the $\mathrm{N}$-terminal Cys exposed after cleavage of $\mathrm{N}$-terminal Met by MetAPs. More recently, it was reported that wild-type RGS2, another R4 subfamily member, has a Gln-2 residue, which is frequently mutated in hypertensive patients to Leu or Arg (13). These mutant RGS2 proteins are processed by either the $\mathrm{Arg} / \mathrm{N}$-end rule pathway or the $\mathrm{Ac} / \mathrm{N}$-end rule pathway. These findings indicate that the two branches of the $\mathrm{N}$-end rule pathway can functionally cooperate and consequently may have a more diverse repertoire of substrate regulation mechanisms.

ATE1 Arg-transferase, the key enzyme in the Arg/N-end rule pathway, is an evolutionarily conserved protein present in all eukaryotes from fungi to animals (14). S. cerevisiae mutants lacking ATE1-encoded Arg-transferase are phenotypically normal (15), whereas ATE1-null mice die at a later stage of embryogenesis (approximately between embryonic day 14.5 [E14.5] and E15.5) due to cardiovascular defects, which resembles various human congenital heart diseases such as ventricular hypoplasia and ventricular septal defects (16). Recently, we reported that the molecular mechanism underlying the characteristic congenital heart disease features of $A T E 1^{-1-}$ mice might be related to the cell-autonomous proliferation defects of cardiomyocytes (17). During those

ISSN: 1976-670X (electronic edition)

Copyright (C) 2016 by the The Korean Society for Biochemistry and Molecular Biology

(c) This is an open-access article distributed under the terms of the Creative Commons Attribution Non-Commercial License (http://creativecommons.org/licenses/by-nc/4.0) which permits unrestricted non-commercial use, distribution, and reproduction in any medium, provided the original work is properly cited. 
studies, however, we frequently observed various gross defects of neurons as well, such as malformation of the brain and cerebral hemorrhages, but their origin and identity were elusive.

In the present study, we demonstrated that in ATE1-null murine embryos, neural-tube genesis is severely defective, and this problem may be the primary cause of embryonic mortality of the mutant mice. ATE1 expression was more prominent in the embryonic brain and spinal cord than in the heart: this pattern overlapped with the migratory path of neural crest cells. Moreover, the ATE1-null embryonic brain showed stabilized RGS proteins, defective G protein signaling, and a higher concentration of NO. Consistent with these biochemical observations, proliferation of $A T E 1^{-1-}$ neuroepithelial cells in the developing primary neural tube was significantly impaired. Taken together, our results suggest that the crucial role of ATE1 in neural tube development may be related to the proper turnover of the RGS4 protein instead of secondary events of blood vessel malformation; the Arg/N-end rule pathway may function as an oxygen sensor and free-radical scavenger.

\section{RESULTS}

Midgestation death and neural abnormality of $A T E 1^{-1-}$ mice Genotyping of litter embryos at E14.5 retrieved from the $A T E^{+/-}$intercross revealed no live homozygous mutants (Fig. $1 \mathrm{~A})$, indicating that the deletion of the ATE1 gene was lethal at midgestation. To examine the timing and pathological nature of the embryonic death of $A T E 1^{-1-}$ mice, we dissected

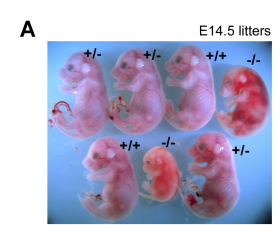

C

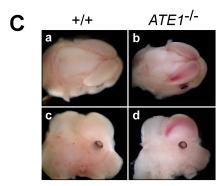

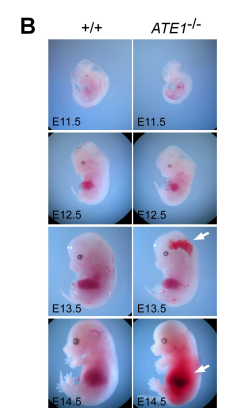

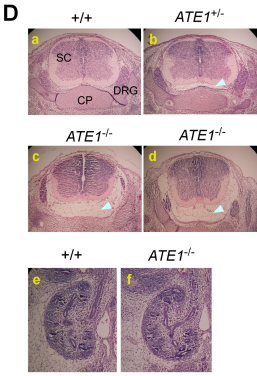

Fig. 1. Characterization of neuronal defects in ATE1-null embryos. (A) Gross morphological features of wild-type and ATE1 ${ }^{-1-}$ mouse embryos at E14.5 from a heterozygous mutant intercross. (B) Gross features of surviving $A T E 1^{-1}$ embryos from E11.5 to E14.5. Arrows indicate internal hemorrhages. (C) Close-up morphological analysis of wild-type $(\mathrm{a}, \mathrm{c})$ and $A T E 1^{-/-}$embryonic brains in transverse and sagittal views. (D) H\&E staining of cross-sections of wild-type and $A T E 1^{-1-}$ spinal cords (near the fourth ventricle) at E12.5 (a, b) and E13.5 (c, d). The ventral part is at the bottom. SC, spinal cord; CP, cartilage primordium; DRG, dorsal root ganglion. Arrowheads indicate cystic space. Kidney slices at E13.5 (f, g) showed no noticeable difference between wild-type and $\mathrm{ATE1}^{-1-}$ murine embryos. morphological characteristics of the embryos produced by timed intercrosses between $A T E 1^{+/-}$individuals at various stages: from E11.5 to E14.5 (Fig. 1B). ATE1 ${ }^{-1-}$ embryos at the stages up to E12.5 were recovered without noticeable morphological or physiological abnormalities. In contrast, $A T E 1^{-1-}$ embryos on E13.5 were distinguishable from their wild-type or heterozygous littermates. Their growth was significantly delayed $(\sim 70 \%$ to $85 \%$ of that in wild-type littermates), and approximately a half of them developed local hemorrhages in lateral ventricles (Fig. 1C). Surviving $A T E 1^{-1-}$ embryos was arrested in size at $\sim$ E13.5 and showed severe pericardial blood leakage; this problem may be the proximal cause of death. ATE $1^{+/-}$mutants did not show any apparent phenotypes, and no gross abnormalities of other major organs were observed.

Because of unexpected defects in $A T E 1^{-1-}$ embryonic brains, we examined histological features of the developing neural tubes from E11.5 to E13.5 by H\&E staining (Fig. 1D). Serial histological sections of E11.5 embryos revealed a normally developing brain and spinal cord. In contrast, at E12.5, while notochord, dorsal root ganglion (DRG), and intermediate layers appeared to be normal, ATE $1^{-/-}$embryos manifested abnormal morphological characteristics such as enlarged cystic space between the spinal cord and the cartilage primordium (Fig. 1D, panels a \& b). The morphological difference was more evident at E13.5 (c \& d), when no significant changes were observed in other organs such as kidneys (e \& f). In mouse embryos, branched nerves start to regulate heart contractions at E12.5; this phenomenon may be linked to our observation that embryonic death occurs at no earlier than $\sim \mathrm{E} 12.5$. Taken together, our results indicate that the lethality of nullification of the ATE1 gene may be mediated by a neuronal abnormality starting at $\sim$ E12.5.

\section{Expression of LacZ from the targeted ATE1 allele in the central nervous system (CNS)}

Expression of a LacZ reporter which is integrated in the ATE1 allele was prominent in the brain and neural tube judging by $\beta$-galactosidase activity (Fig. 2A), which was consistent with ATE1 mRNA expression from in situ hybridization analysis (16). We also examined expression of ATE1 in histological slices using the anti-RGS4 antibody (Fig. 2B). The RGS4 protein is extremely short-lived in the wild-type background but is stabilized in ATE1-null animals. Therefore, ATE1 ${ }^{-1-}$ mice can serve as a rare experimental model for monitoring the RGS4 expression. At E11.5, when the neural folds still had not closed, the RGS4 protein was expressed at the boundary between the non-neural ectoderm and neural plates (Fig. 2B). As the embryo grew, the RGS4-positive cells migrated ventrally and cranially into the DRG, notochord, and sympathetic ganglia, which are also the destinations of migrating neural crest cells. This finding suggests that degradation of the RGS4 protein by ATE 1 is closely associated with the migration or differentiation of neural crest cells during embryogenesis. In 
A

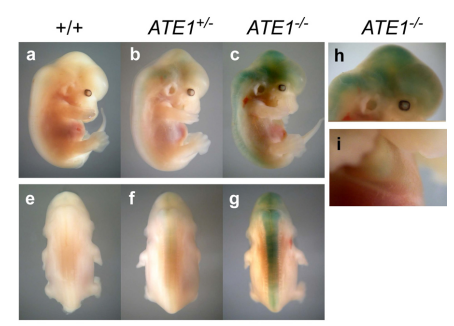

B

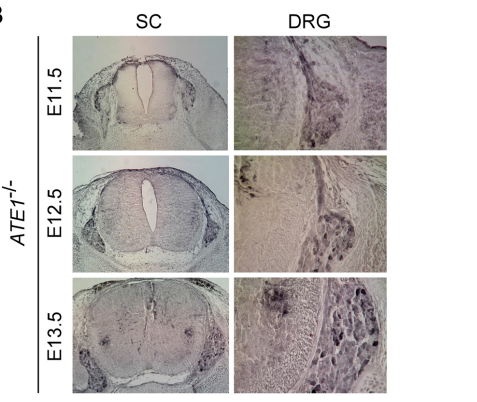

Fig. 2. ATE1 is expressed in the CNS and migrates during neural tube development. (A) LacZ staining of whole embryos of wildtype $(\mathrm{a}, \mathrm{e}), \operatorname{ATE1}^{+1-}(\mathrm{b}, \mathrm{f})$, and $\operatorname{ATE}^{-1-}(\mathrm{c}, \mathrm{g})$ mice at E13.5. Enlarged views of the brain (h) and heart (i) of $A T E 1^{-1-}$ embryos. (B) Immunostaining for RGS4 on cross-sections of ATE1 ${ }^{-l}$ embryonic tissues indicates that RGS4 expression was highly colocalized with the migration path of neural crest cells, which includes ventral migration to DRG and other migratory ends.

addition, because neural crest cells migrate into the heart and vessels, it is possible that impaired migration of neural crest cells may lead to the cardiovascular defects in $A T E 1^{-1-}$ embryos.

\section{Stabilized RGS proteins in ATE1-null mice and reduced activities of downstream effectors}

Previously, we identified a set of RGS proteins (RGS4, RGS5, and RGS16) as the primary in vivo substrates of murine ATE1 (12). Nonetheless, our understandings were mainly obtained by means of in vitro-generated RGS proteins and transiently transfected RGS proteins in ATE1-null MEFs because of a lack of good antibodies. Using recently produced monoclonal antibodies $(17,18)$, we found that the levels of endogenous RGS4 dramatically increased in $A T E 1^{-1-}$ whole embryos and embryonic brains (Fig. 3A and 3B). The RGS4 protein was virtually undetectable in wild-type and $A T E 1^{+/-}$littermate controls. The effect of ATE1 inactivation on RGS16 levels was not as striking as that on RGS4, but the RGS16 protein was also stabilized in $A T E 1^{-1-}$ mice. We also found that the amount of RGS4 was greater in the brain than in the heart (data not shown). Quantitative RT-PCR analysis of total RNA showed no significant difference between the mutants and wild-type embryonic brains at E13.5; this finding indicates that downregulation of RGS proteins takes place at the posttran-

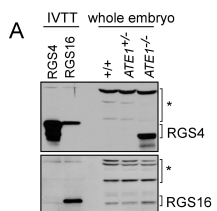

B

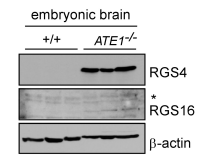

E

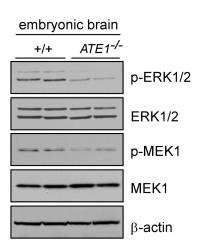

C

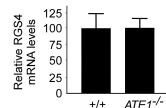

$\mathrm{F}$
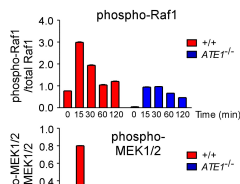

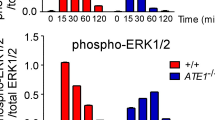

Fig. 3. ATE1 is required for degradation of RGS proteins and GPCR signaling. (A) Immunoblotting for RGS4 and RGS16 in extracts of wild-type and $A T E 1^{-1-}$ whole embryos. In vitro transcribed and translated (IVTT) RGS4 and RGS5 proteins (25) were coloaded as positive controls. Asterisks indicate nonspecific signals. (B) Same as (A), except that embryonic brain extracts were used. (C) Quantitative RT-PCR analysis of RNA from wild-type and ATE $^{-\zeta-}$ embryonic brains (E13.5). (D) Immunoblotting analysis of MAPK-related downstream effectors of RGS proteins. Wild-type and $A T E 1^{-1-}$ embryonic brains were subjected to immunoblotting with various antibodies. (E) The serum starvation/stimulation assay of the MAPK pathway using primary MEFs isolated from wild-type and $A T E 1^{-l-}$ embryos at E13.5. (F) Activation profiles of the kinases quantified using the data from panel $(E)$.

scriptional stage (Fig. 3C). These results point to a strong spatiotemporal correlation between protein arginylation and its substrate, RGS4, in the developing murine brain.

Considering the role of RGS proteins as negative regulators in $G$ protein-mediated signaling as well as the prominent expression of ATE1 in the brain and spinal cord, we tested whether the G protein-coupled receptor (GPCR) signaling pathway in the ATE1-mutant brain is defective. To this end, we first examined the downstream kinase activity of the GPCR signaling pathway by an immunoblot assay using embryonic brains (Fig. 3D). Previously, we reported that the activity of ERK1 and ERK2-downstream effectors of $G_{q}$ and $G_{i}$ signaling pathways - is impaired in the whole embryo (19). In embryonic brain extracts, phosphorylated forms of ERK1/2 and MEK1 were significantly downregulated, while total kinase levels were comparable to wild-type levels. To further characterize the effects of ATE1 on MAPK components, we examined responsiveness of kinase activities to serum stimulation in primary culture of wild-type and $A T E 1^{-1-}$ MEFs (Fig. 3E). Primary MEFs were harvested at multiple time points after $24 \mathrm{~h}$ serum starvation and subsequent $10 \%$ serum activation, and kinase activities were measured by immunoblotting using activated kinases-specific antibodies. The activities enhanced by serum stimulation were observed as early as $15 \mathrm{~min}$ later. Nevertheless, significantly decreased levels of active forms of RAF1, MEK1, and ERK1/2 were 
detected in ATE1 ${ }^{-1-}$ MEFs compared to wild-type MEFs (Fig. $3 F)$, suggesting that the ATE1-RGS pathway tightly regulates the MEK1-ERK1/2 pathway. Taken together, these findings are consistent with another report showing that RGS proteins downregulate $\mathrm{G}$ protein signaling in primary cardiomyocytes (19) and suggest that $G_{q}$ signaling may be reduced in ATE1-null cells because of stabilization of negative regulators of this signaling pathway (RGS proteins) in the brain.

\section{Defective neuronal-cell proliferation in the ATE1-null embryonic brain}

Based on the finding that MAPK signaling in the ATE1 ${ }^{-1-}$ brain was reduced and RGS-regulated $\mathrm{G}_{\mathrm{q}}$ and $\mathrm{G}_{\mathrm{i}}$ signaling pathways were defective, we hypothesized that the aforementioned neural defects of $A T E 1^{-1-}$ embryos originate from decreased proliferation. In addition, although the formation and shaping of the neural plate of $A T E 1^{-1-}$ embryos appeared to be normal, the closure of the neural groove to from the neural tube was slower than that of wild-types. For quantitative comparison, we examined the rates of neural-cell proliferation in the $A T E 1^{-1-}$ neural tube by immunostaining for $\mathrm{H} 3 \mathrm{P}, \mathrm{a}$ mitosis marker, from E11.5 to E13.5 (Fig. 4A). In wild-type embryos, H3P-positive cells were usually observed at earlier stages (E11.5 and E12.5), but there were little mitotic cells at E13.5. ATE $1^{-1-}$ embryos showed a markedly reduced number of cells with relative $\mathrm{H} 3 \mathrm{P}$-positive staining in the neural tube (especially in the ventricular layers) in comparison with those in wild-type littermates (Fig. 4B). The mitotic index of wild-type neuroepithelial cells was $13.3 \%$, while in ATE1 $1^{-1-}$ mutants, this index was significantly reduced: $3.5 \%$. On E12.5, the difference was smaller: $5.9 \%$ in the wild type versus $3.1 \%$ in $A T E 1^{-1-}$ mice (Fig. 4C). The size of the lumen of the neural tube appeared to be slightly enlarged in mutant embryos on E11.5 and E12.5. To date, not many human genes associated with neural-tube defects have been identified although these problems are prevalent among human birth defects. Our study indicates that the neural-tube defects observed in $A T E 1^{-1-}$ mice result from alterations in proliferation of the neuroepithelial cells, which eventually differentiate into the numerous types of neurons and glial cells in the body.

It is notable that at E11.5, no mortality or changes in gross morphological features were seen in $A T E 1^{-1-}$ embryos, indicating that the impaired mitosis of neuroepithelial cells at the earlier stage may account for the defective neural morphogenesis and, ultimately, for the fetal death. We also studied the cell death by the TUNEL assay to determine whether abnormally increased apoptosis results in the decreased cellular proliferation in the ATE1 $1^{-1-}$ neural tubes from E12.5 to E13.5. Only a few neural cells exhibited apoptotic signals during the stages preceding the global necrosis of $A T E 1^{-1-}$ embryos, indicating negligible cell death by apoptosis, and we saw no indication that the increase in cell death contributed to this phenotype (data not shown). Therefore, protein arginylation by ATE1 may perform an essential function in proliferation of neuronal cells, not in apoptotic cell death.

The arginylation branch of the mammalian $\mathrm{N}$-end rule pathway is implicated in oxygen concentration-dependent degradation of target substrates (20). In yeast, $\mathrm{N}$-terminal Cys is a stabilizing (nonarginylated) residue (21). When we measured the concentration of NO metabolites, there were significantly larger amounts of nitrites and nitrates both in whole embryos and in embryonic brains of $A T E 1^{-1-}$ mice. This result is consistent with our previous finding in MEFs that RGS proteins require a series of $\mathrm{N}$-terminal modifications, including oxidation of $\mathrm{N}$-terminally exposed Cys-2 (12). This is a consequence of competition among the post-translational modifications at the Cys-2 including acetylation and palmitoylation, and probably, the major determining step at the crossroads to the arginylation and acetylation branch of the $\mathrm{N}$-end rule pathway. The $\mathrm{Arg} / \mathrm{N}$-end rule pathway may function as a licensing step to regulate GPCR signaling depending on the cellular oxygen concentration, which also varies among developmental stages. Although the underlying
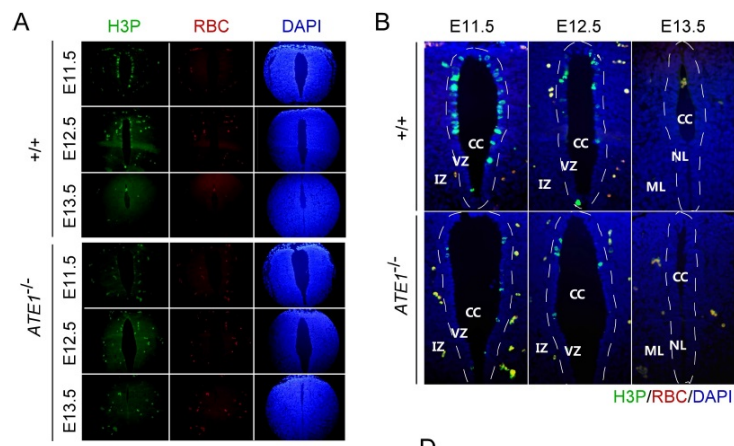

C

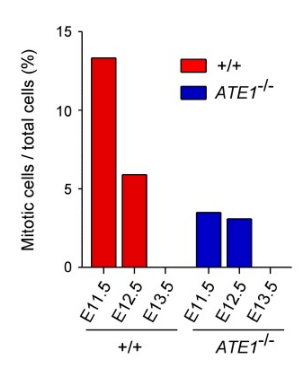

D
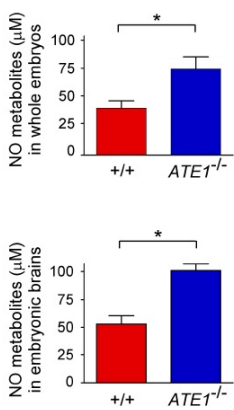

Fig. 4. ATE1-null embryos show impaired proliferation of neuroepithelial cells. (A) Immunostaining for $\mathrm{H} 3 \mathrm{P}$ on cross-sections of wild-type and $A T E 1^{-1-}$ neural tubes at E11.5, E12.5, and E13.5 (counterstaining with DAPI). Red blood cells (RBC) showed nonspecific signals. (B) Merged images of neural tube areas from panel (A). (C) Quantification of mitotic cells in the neuroepithelium. (D) Amounts of $\mathrm{NO}$ metabolites in wild-type and ATE1 ${ }^{-1}$ whole embryos (top) and embryonic brains (bottom) at E13.5 were measured by the Griess assay. Values represent means \pm SD $(\mathrm{n}=$ 3). ${ }^{* P}<0.05$ (two-tailed t-test). 
molecular mechanisms have yet to be determined, these data strongly suggest that the ATE1-RGS circuit is implicated in normal development of the neural tube during embryogenesis.

\section{DISCUSSION}

In the $\mathrm{N}$-end rule-dependent proteolytic system, the $\mathrm{N}$-terminal Arg is post-translationally attached by ATE1 Arg-transferase and functions as a degradation determinant. We previously reported that $A T E 1^{-1-}$ mice die because of various cardiovascular defects during embryogenesis (19) although the molecular etiology is still unknown. Our data in this study collectively indicate that ATE1 is also necessary for neurogenesis, and that the CNS phenotype observed in ATE1-null embryos may be due to a mitotic aberration of neuronal cells. Neuronal cells undergo mitosis when they are located lumenally; this activity is a part of closure of hollow neural tubes. Therefore, the cellular-proliferation defects in $A T E 1^{-1-}$ mice may lead to malformation of the ventricular zone accompanied with various CNS alterations. Because the neural stem cell migration of $A T E 1^{-1-}$ embryos was normal, the delayed closure of the neural groove of $A T E 1^{-1-}$ mice appeared not due to the defected paracrine signal of the nearby neural crest cells. Functioning of ATE1 is necessary for degradation of RGS proteins and for proper activation of GPCR signaling pathways probably in a cell-autonomous manner. Our findings indicate that neuroepithelial cells and the cerebral ventricular system may account for the embryonic death of $A T E 1^{-1-}$ mice. Whether the cardiac defects and neural-tube defects in $A T E 1^{-1-}$ embryos are independent or causally linked has yet to be identified, and this knowledge will elucidate not only the physiological function of ATE1 in mammals but the connection of GPCR signaling with migration of neural crest cells during embryonic development. A recent study on adult ATE1-null mice (created by the Cre-Lox method) showed, however, a strikingly broad range of phenotypes (22), indicating that the ATE1-RGS-G protein circuit may be involved in a greater variety of essential biological pathways than previously thought. A likely explanation would be that the reduced availability of $\mathrm{O}_{2}$ at the developing neural tube areas may be critical for stabilizing the RGS proteins, GPCR signaling, and proper proliferation of neuroepithelium under normal physiological conditions. Therefore, the oxygensensing mechanism by the $\mathrm{Arg} / \mathrm{N}$-end rule pathway is the additional layers of the regulatory steps of $\mathrm{G}$ protein signaling to maintain the proper development of the CNS.

\section{MATERIALS AND METHODS}

\section{Mice}

Exons 1 through 3 of the ATE 1 gene were disrupted in CJ7 embryonic stem cells as previously described (16). Embryos at E10.5 to E17.5 were obtained from intercrosses of heterozygous mice in the $129 \mathrm{SvEv} / \mathrm{C} 57 \mathrm{BL} / 6$ (mixed) genetic background. The presence of a vaginal plug after overnight mating was regarded as E0.5. Genotyping of the yolk sac or tail DNA of each embryo was performed using a standard polymerase chain reaction.

\section{Histological analysis and $\beta$-galactosidase staining}

For histological analysis, embryos were fixed overnight at $4^{\circ} \mathrm{C}$ in $4 \%$ paraformaldehyde (Fisher Scientific, Hampton, $\mathrm{NH}$ ) in ice-cold phosphate-buffered saline (PBS). The specimens were incubated with $70 \%$ ethanol, dehydrated, embedded in paraffin wax, and sectioned transversely into slices $7 \mu \mathrm{m}$ thick, followed by staining with hematoxylin and eosin (H\&E). To detect the activity of $\beta$-galactosidase on the slices, we stained the fixed samples overnight at $37^{\circ} \mathrm{C}$ in an X-gal solution $(1.3$ $\mathrm{mg} / \mathrm{ml}$ potassium ferrocyanide, $1 \mathrm{mg} / \mathrm{ml}$ potassium ferricyanide, $0.3 \%$ Triton X-100, $1 \mathrm{mM}[\mathrm{mmol} / \mathrm{L}] \mathrm{MgCl}_{2}, 150 \mathrm{mM}$ $\mathrm{NaCl}$, and $1 \mathrm{mg} / \mathrm{ml}$ 4-chloro-5-bromo-3-indolyl- $\beta$-galactoside [X-gal, Roche Applied Science, Indianapolis, IN] in PBS [pH 7.4]).

\section{In vivo proliferation assay}

The neural-tube slices were incubated with an antiphosphohistone H3 (H3P) antibody (Upstate, Charlottesville, VA; 1:100 dilution), followed by a fluorescein isothiocyanate (FITC)-conjugated anti-rabbit IgG antibody (Jackson Immunoresearch, West Grove, PA). All the antibodies were incubated with the slices for $1 \mathrm{~h}$ at room temperature in $1 \%$ goat serum in PBST (PBS with 10\% Tween 20), and the secondary antibody was diluted 1 to 200 (23). The cells were counterstained with 4',6-diamidino-2-phenylindole (DAPI, Vector Laboratories, Burlingame, CA) to visualize the nuclei.

\section{Immunotechniques}

For immunoblotting analysis, whole embryos or embryonic brains at E13.5 or E14.5 were isolated in lysis buffer $(20 \mathrm{mM}$ HEPES [pH 7.5], $150 \mathrm{mM} \mathrm{KCl}, 10 \%$ glycerol, and $0.1 \mathrm{mM}$ EDTA). A soluble fraction was separated by two-step centrifugation $(9,000 \times g$ and $100,000 \times$ g), subjected to electrophoresis, blotted to a polyvinylidene fluoride membrane, and probed with an anti-RGS4 or anti-RGS16 antibody (17, 18), followed by reprobing with an anti-actin antibody (Sigma-Aldrich).

\section{Examination of MAPK pathway effectors}

To analyze activities of the MAPK pathway, we subjected total extracts from wild-type and $A T E 1^{-1-}$ brains and primary mouse embryonic fibroblasts (MEFs) to immunoblotting with antibodies against ERK1/2, MEK1/2, RAF1, their phosphorylated forms (Cell Signaling Technology, Beverly, MA), Gaq (Santa Cruz Biotechnology, Dallas, TX), and actin (Sigma-Aldrich). Quantification was performed using the ImageJ software (version 1.46r, National Institutes of Health, Bethesda, MD). 


\section{Quantification of NO metabolites}

For measurement of nitrite $\left(\mathrm{NO}_{2}{ }^{-}\right)$and nitrate $\left(\mathrm{NO}_{3}{ }^{-}\right)$levels in whole embryos and embryonic brains, tissue samples from wild-type and $A T E 1^{-1-}$ mice were homogenized in a buffer consisting of $25 \mathrm{mM}$ Tris-HCl (pH 7.5), $0.5 \mu \mathrm{M}$ EDTA, and 0.5 $\mu \mathrm{M}$ EGTA and were centrifuged at 14,000 $\times \mathrm{g}$ for $15 \mathrm{~min}$. Nitrite was assayed using the Griess reaction (24). Nitrate content was determined after conversion of nitrate to nitrite by means of Aspergillus nitrate reductase (Sigma-Aldrich).

\section{ACKNOWLEDGEMENTS}

This work was supported by the grants of the Health Industry Development Institute (HI14C0202, HI14C2036 to M.J.L.) and the National Research Foundation of Korea (2013R1A1A20 59793, 2016R1C1B2011367 to J.H.L., 2013R1A2A2A01014170 to Y.T.K., 2016R1A2B2006507 to M.J.L.). This research was also supported by the Brain Research Program (2016M 3C7A1913895) and the Creative-Pioneering Researchers Program of SNU.

\section{REFERENCES}

1. Bachmair A, Finley D and Varshavsky A (1986) In vivo half-life of a protein is a function of its amino-terminal residue. Science 234, 179-186

2. Lee JH, Jiang $Y$, Kwon YT and Lee MJ (2015) Pharmacological Modulation of the N-End Rule Pathway and its Therapeutic Implications. Trends Pharmacol Sci 36, 782-797

3. Varshavsky A (1996) The N-end rule: functions, mysteries, uses. Proc Natl Acad Sci U S A 93, 12142-12149

4. Kwon YT, Kashina AS and Varshavsky A (1999) Alternative splicing results in differential expression, activity, and localization of the two forms of arginyl-tRNAprotein transferase, a component of the $\mathrm{N}$-end rule pathway. Mol Cell Biol 19, 182-193

5. Kwon YT, Reiss Y, Fried VA et al (1998) The mouse and human genes encoding the recognition component of the $\mathrm{N}$-end rule pathway. Proc Natl Acad Sci U S A 95, 7898-7903

6. Tasaki T, Mulder LC, Iwamatsu A et al (2005) A family of mammalian E3 ubiquitin ligases that contain the UBR box motif and recognize N-degrons. Mol Cell Biol 25, 7120-7136

7. Shrader TE, Tobias JW and Varshavsky A (1993) The $\mathrm{N}$-end rule in Escherichia coli: cloning and analysis of the leucyl, phenylalanyl-tRNA-protein transferase gene aat. J Bacteriol 175, 4364-4374

8. Tobias JW, Shrader TE, Rocap G and Varshavsky A (1991) The $\mathrm{N}$-end rule in bacteria. Science 254, 1374-1377

9. Erbse A, Schmidt R, Bornemann T et al (2006) ClpS is an essential component of the $\mathrm{N}$-end rule pathway in Escherichia coli. Nature 439, 753-756
10. Kaji H, Novelli GD and Kaji A (1963) A Soluble Amino Acid-Incorporating System From Rat Liver. Biochim Biophys Acta 76, 474-477

11. Hu RG, Sheng J, Qi X, Xu Z, Takahashi TT and Varshavsky A (2005) The $\mathrm{N}$-end rule pathway as a nitric oxide sensor controlling the levels of multiple regulators. Nature 437, 981-986

12. Lee MJ, Tasaki T, Moroi $\mathrm{K}$ et al (2005) RGS4 and RGS5 are in vivo substrates of the $\mathrm{N}$-end rule pathway. Proc Natl Acad Sci U S A 102, 15030-15035

13. Park SE, Kim JM, Seok OH et al (2015) Control of mammalian $\mathrm{G}$ protein signaling by $\mathrm{N}$-terminal acetylation and the $\mathrm{N}$-end rule pathway. Science 347, 1249-1252

14. Hu RG, Brower CS, Wang $\mathrm{H}$ et al (2006) Arginyltransferase: specificity, putative substrates, bidirectional promoter, and splicing-derived isoforms. J Biol Chem 281, 32559-32573

15. Balzi E, Choder M, Chen WN, Varshavsky A and Goffeau A (1990) Cloning and functional analysis of the arginyltRNA-protein transferase gene ATE1 of Saccharomyces cerevisiae. J Biol Chem 265, 7464-7471

16. Kwon YT, Kashina AS, Davydov IV et al (2002) An essential role of $\mathrm{N}$-terminal arginylation in cardiovascular development. Science 297, 96-99

17. Derrien A, Zheng B, Osterhout JL et al (2003) Srcmediated RGS16 tyrosine phosphorylation promotes RGS16 stability. J Biol Chem 278, 16107-16116

18. Tamirisa P, Blumer KJ and Muslin AJ (1999) RGS4 inhibits G-protein signaling in cardiomyocytes. Circulation 99, 441-447

19. Lee MJ, Kim DE, Zakrzewska A et al (2012) Characterization of arginylation branch of $\mathrm{N}$-end rule pathway in G-protein-mediated proliferation and signaling of cardiomyocytes. J Biol Chem 287, 24043-24052

20. Sriram S, Lee JH, Mai BK et al (2013) Development and characterization of monomeric $\mathrm{N}$-end rule inhibitors through in vitro model substrates. J Med Chem 56, 2540-2546

21. Turner GC and Varshavsky A (2000) Detecting and measuring cotranslational protein degradation in vivo. Science 289, 2117-2120

22. Brower CS and Varshavsky A (2009) Ablation of arginylation in the mouse $\mathrm{N}$-end rule pathway: loss of fat, higher metabolic rate, damaged spermatogenesis, and neurological perturbations. PLoS One 4, e7757

23. Hu L, Su P, Yan K et al (2015) Knockdown of microtubule actin crosslinking factor 1 inhibits cell proliferation in MC3T3-E1 osteoblastic cells. BMB Rep 48, 583-588

24. Ju SM, Youn GS, Cho YS, Choi SY, and Park J (2015) Celastrol ameliorates cytokine toxicity and pro-inflammatory immune responses by suppressing NF- $\mathrm{KB}$ activation in RINm5F beta cells. BMB Rep 48, 172-177

25. Jiang Y, Pore SK, Lee JH et al (2013) Characterization of mammalian $\mathrm{N}$-degrons and development of heterovalent inhibitors of the $\mathrm{N}$-end rule pathway. Chem Sci 4, 3339-3346 